\title{
Fuzzy Economic Order Quantity (FEOQ) Model with Units Lost Due to Deterioration
}

\author{
M.Pattnaik ${ }^{1, *}$ \\ ${ }^{1}$ Department of Business Administration, Utkal University, Bhubaneswar,India.
}

Received 4 November 2013; Accepted 17 May 2014

Editor: David G. Yu

\begin{abstract}
This model investigates the instantaneous fuzzy economic order quantity model by allocating the percentage of units lost due to deterioration in an on-hand inventory by framing variable ordering cost. The objective is to maximize the fuzzy net profit so as to determine the order quantity, the cycle length and number of units lost due to deterioration in fuzzy decision space. For any given number of replenishment cycles the existence of a unique optimal replenishment schedule are proved and mathematical model is developed to find some important characteristics for the concavity of the fuzzy net profit function. Numerical examples are provided to illustrate the results of proposed model which benefit the retailer and this policy is important, especially for wasting of deteriorating items. Finally, sensitivity analyses of the fuzzy optimal solution with respect to the major parameters are also studied.
\end{abstract}

Keywords Keywords- Fuzzy, FEOQ, Units lost, Deterioration, Variable ordering cost

DOI: $10.19139 /$ soic.v2i3.40

\section{Introduction}

Most of the literature on inventory control and production planning has dealt with the assumption that the demand for a product will continue infinitely in the future either in a deterministic or in a stochastic fashion. This assumption does not always hold true. Inventory management plays a crucial role in businesses since it can help companies reach the goal of ensuring prompt delivery, avoiding shortages, helping sales at competitive prices and so forth. The mathematical modeling of

\footnotetext{
${ }^{*}$ Correspondence to: Dept. of Business Administration, Utkal University, Bhubaneswar, India751004, Email: monalisha_1977@yahoo.com
}

ISSN 2310-5070 (online) ISSN 2311-004X (print)

Copyright (c) 2014 International Academic Press 
real-world inventory problems necessitates the simplification of assumptions to make the mathematics flexible. However, excessive simplification of assumptions results in mathematical models that do not represent the inventory situation to be analyzed.

Many models have been proposed to deal with a variety of inventory problems. The classical analysis of inventory control considers three costs for holding inventories. These costs are the ordering cost, carrying cost and shortage cost. The classical analysis builds a model of an inventory system and calculates the EOQ (economic order quantity) which minimize these three costs so that their sum is satisfying minimization criterion. One of the unrealistic assumptions is that items stocked preserve their physical characteristics during their stay in inventory. Items in stock are subject to many possible risks, e.g. damage, spoilage, dryness; vaporization etc., those results decrease of usefulness of the original one and a cost is incurred to account for such risks. In the whole production system production function is the mid between the procurement function and physical distribution function. Other two functions are not processing in terms of production only they are facilitating for the smooth functioning and cost effecting of the production system in competitive advantage but production function processes to produce the finished products. So inventory plays a significant role in smooth functioning of the production function in a supply chain management. The physical characteristics of stocked items dictate the nature of inventory policies implemented to manage and control in production system. The question is how reliable are the EOQ models when items stocked deteriorate one time.

Many models have been proposed to deal with a variety of inventory problems. Comprehensive reviews of inventory models can be found in [4, 11, 27, 20] introduced a single item EOQ model with two constraints. This model considers a continuous review, using fuzzy arithmetic approach to the system cost for instantaneous production process. In traditional inventory models it has been common to apply fuzzy on demand rate, production rate and deterioration rate, whereas applying fuzzy arithmetic in system cost usually ignored in [18]. From practical experience, it has been found that uncertainty occurs not only due to lack of information but also as a result of ambiguity concerning the description of the semantic meaning of declaration of statements relating to an economic world. The fuzzy set theory was developed on the basis of non-random uncertainties. In [26], Vujosevic et al. introduced the EOQ model where inventory system cost is fuzzy. Mahata and Goswami [9] then presented production lot size model with fuzzy production rate and fuzzy demand rate for deteriorating items where permissible delay in payments are allowed. Roy and Maiti [16] presented fuzzy EOQ model with demand dependent unit cost under limited storage capacity. In Tripathy and Pattnaik [22] presented an optimal inventory policy with reliability consideration and instantaneous receipt under imperfect production process. Later, they also investigated fuzzy EOQ model with reliability consideration in instantaneous 
production plan and eveloped fuzzy entropic order quantity model for perishable items under two component demand and discounted selling price, where entropic means the amount of the disorder in the production system [24]. In [13], Pattnaik discussed the fuzzy EOQ model with demand dependent unit price and variable setup cost, For this reason, this model considers the same by introducing the holding cost and ordering cost as with allowing promotion and wasting the percentage of the fuzzy numbers. Sahoo and Pattnaik [17] developed linear programming problem and post optimality analyses in fuzzy space with case study applications. The model provides an approach for quantifying the benefits of nonrandom uncertainty which can be substantial, and should be reflected in fuzzy arithmetic system cost.

Product perishability is an important aspect of inventory control. Deterioration in general, may be considered as the result of various effects on stock, some of which are damage, decay, decreasing usefulness and many more. While kept in store fruits, vegetables, food stuffs etc. suffer from depletion by decent spoilage. Decaying products are of two types. Product which deteriorate from the very beginning and the products which start to deteriorate after a certain time. Lot of articles is available in inventory literature considering deterioration. Interested readers may consult the survey model of Goyal and Gunasekaran [3] and Raafat [15] surveyed for perishable items to optimize the EOQ model. The EOQ inventory control model was introduced in the earliest decades of this century and is still widely accepted by many industries today. Tripathy and Pattnaik [23] derived different types of typical deterministic EOQ models in crisp and fuzzy decision space.

Comprehensive reviews of inventory models under deterioration can be found in [1]. In previous deterministic inventory models, many are developed under the assumption that demand is either constant or stock dependent for deteriorated items. Jain and Silver [8] developed a stochastic dynamic programming model presented for determining the optimal ordering policy for a perishable or potentially obsolete product so as to satisfy known time-varying demand over a specified planning horizon. They assumed a random lifetime perishability, where, at the end of each discrete period, the total remaining inventory either becomes worthless or remains usable for at least the next period. Mishra [10] explored the inventory model for time dependent holding cost and deterioration with salvage value where shortages are allowed. Gupta and Gerchak [4] examined the simultaneous selection product durability and order quantity for items that deteriorate over time. Their choice of product durability is modeled as the values of a single design parameter that effects the distribution of the time-to-onset of deterioration (TOD) and analyzed two scenarios; the first considers TOD as a constant and the store manager may choose an appropriate value, while the second assumes that TOD is a random variable. Hariga [5] considered the effects of inflation and the time-value of money with the assumption of two inflation rates 
rather than one, i.e. the internal (company) inflation rate and the external (general economy) inflation rate. Hariga [7] argued that the analysis of Bose et al. [1] contained mathematical errors for which he proposed the correct theory for the problem supplied with numerical examples. Padmanavan and Vrat [12] presented an EOQ inventory model for perishable items with a stock dependent selling rate. They assumed that the selling rate is a function of the current inventory level and the rate of deterioration is taken to be constant. The most recent work found in the literature is that of Hariga [6] who extended his earlier work by assuming a time-varying demand over a finite planning horizon. Goyal et al. [2] and Shah [19] explored the inventory models for deteriorating items.

Furthermore, retailer promotional activity has become more and more common in today's business world. For example, Wall Mart and Costco often try to stimulate demand for specific types of electric equipment by offering price discounts; clothiers Baleno and NET make shelf space for specific clothes items available for longer periods; McDonald's and Burger King often use coupons to attract consumers. Other promotional strategies include free goods, advertising, and displays and so on. The promotion policy is very important for the retailer. How much promotional effort the retailer makes has a big impact on annual profit. Residual costs may be incurred by too many promotions while too few may result in lower sales revenue. Tsao and Sheen [25] discussed dynamic pricing, promotion and replenishment policies for a deteriorating item under permissible delay in payment. Salameh et al. [18] studied an EOQ inventory model in which it assumes that the percentage of on-hand inventory wasted due to deterioration is a key feature of the inventory conditions which govern the item stocked. The effect of deteriorating items on the instantaneous profit maximization replenishment model under promotion is considered in this model. The market demand may increase with the promotion of the product over time when the units lost due to deterioration. In the existing literature about promotion it is assumed that the promotional effort cost is a function of promotion. Tripathy et al. [23] investigated an optimal EOQ model for deteriorating items with promotional effort cost. Pattnaik [14] explored the effect of promotion in fuzzy optimal replenishment model with units lost due to deterioration. Hence Pattnaik [13] developed many instantaneous EOQ models and fuzzy EOQ models which are incorporated with promotional effort cost, fixed ordering cost, variable ordering cost and units lost due to deterioration. All mentioned above inventory literatures with deterioration and percentage of on-hand inventory due to deterioration have the basic assumption that the retailer owns a storage room with optimal order quantity. This model establishes and analyzes the fuzzy inventory model under profit maximization which extends the classical economic order quantity (EOQ) model. An efficient EOQ does more than just reduce cost. It also creates revenue for the retailer and the manufacturer. The evolution of the FEOQ (fuzzy economic order quantity) model concept tends toward revenue and demand focused strategic 
formation and decision making in business operations. Evidence can be found in the increasingly prosperous revenue and yield management practices and the continuous shift away from supply-side cost control to demand-side revenue stimulus. This model introduces a modified fuzzy EOQ model in which it assumes that a percentage of the on-hand inventory is wasted due to deterioration. There is hidden cost not account for when modeling inventory cost. This model studies the problem of promotion for a deteriorating item subject to loss of these deteriorated units. This model postulates that measuring the behavior of production systems may be achievable by incorporating the idea of retailer in making optimum decision on replenishment with wasting the percentage of on-hand inventory due to deterioration and then compares the optimal results with none wasting the percentage of on-hand inventory due to deterioration traditional model. This model addresses the problem by proposing an inventory model under promotion by assuming that the units lost due to deterioration of the items. In this model, promotional effort and replenishment decision are adjusted arbitrarily upward or downward for profit maximization model in response to the change in market demand within the planning horizon. The objective of this model is to determine the optimal time length, optimal units lost due to deterioration, the promotional effort and the replenishment quantity with variable ordering cost so that the net profit is maximized in an instantaneous replenishment fuzzy EOQ model and the numerical analysis show that an appropriate promotion policy can benefit the retailer and that promotion policy is important in fuzzy space, especially for deteriorating items. Finally, sensitivity analysis of the optimal solution with respect to the major parameters are also studied to draw the managerial insights.

In recent years, companies have started to recognize that a tradeoff exists between product varieties in terms of quality of the product for running in the market smoothly. In the absence of a proper quantitative model to measure the effect of product quality of the product, these companies have mainly relied on qualitative judgment. The model tackles to investigate the effect of the wasting the percentage of on-hand inventory due to deterioration for obtaining the optimum average payoff and the optimal values of the policy variables. The problem consists of the optimization of fuzzy EOQ model, taking into account the conflicting payoffs of the different decision makers involved in the process. A policy iteration algorithm is designed and optimum solution is obtained through LINGO 13.0 version software. In order to make the comparisons equitable a particular evaluation function is suggested. This model postulates that measuring the behavior of production systems may be achievable by incorporating the idea of retailer in making optimum decision on replenishment with wasting the percentage of on-hand inventory due to deterioration with dynamic ordering cost in fuzzy decision space and then compares the optimal results with none wasting percentage of on-hand inventory due to deterioration traditional model. The major assumptions used in the above research articles are summarized in Table I. 
Table I. Summary of the Related Researches

\begin{tabular}{|c|c|c|c|c|c|c|c|c|}
\hline $\begin{array}{c}\text { Author(s) } \\
\text { and } \\
\text { published Year }\end{array}$ & $\begin{array}{c}\text { Structure } \\
\text { of the } \\
\text { model }\end{array}$ & Demand & $\begin{array}{c}\text { Demand } \\
\text { patterns }\end{array}$ & Deterioration & $\begin{array}{c}\text { Ordering } \\
\text { cost }\end{array}$ & Planning & $\begin{array}{c}\text { Units Lost due } \\
\text { Deterioration }\end{array}$ & Model \\
\hline $\begin{array}{c}\text { Hariga[7] } \\
(1994)\end{array}$ & $\begin{array}{c}\text { Crisp } \\
\text { (EOQ) }\end{array}$ & Time & $\begin{array}{c}\text { Non- } \\
\text { stationary }\end{array}$ & Yes & Constant & Finite & No & Cost \\
\hline $\begin{array}{c}\text { Tsao et al.[25] } \\
(2008)\end{array}$ & $\begin{array}{c}\text { Crisp } \\
\text { (EOQ) }\end{array}$ & $\begin{array}{c}\text { Time and } \\
\text { Price }\end{array}$ & $\begin{array}{c}\text { Linear and } \\
\text { Decreasing }\end{array}$ & Yes & Constant & Finite & No & Profit \\
\hline $\begin{array}{c}\text { Present } \\
\text { model }\end{array}$ & $\begin{array}{c}\text { Fuzzy } \\
\text { (FEOQ) }\end{array}$ & $\begin{array}{c}\text { Constant } \\
\text { (Deterministic) }\end{array}$ & Constant & $\begin{array}{c}\text { Yes } \\
\text { (Wasting) }\end{array}$ & Variable & Finite & Yes & Profit \\
\hline
\end{tabular}

The remainder of the model is organized as follows: in Section 2 assumptions and notations are provided for the development of the model. The mathematical formulation is developed in Section 3. In Section 4 the fuzzy mathematical model is derived. The solution procedure is given in Section 5. In Section 6, numerical example is presented to illustrate the development of the model. The sensitivity analysis are studied in Section 7 to observe the changes in the optimal solution for change in one major parameter. Finally in Section 8 the summary and the concluding remarks are explained.

\section{Assumptions and Notations}

$r:$ Consumption rate,

$t_{c}:$ Cycle length,

$h$ : Holding cost of one unit for one unit of time,

$H C(q)$ : Holding cost per cycle,

$c:$ Purchasing cost per unit,

$P_{s}:$ Selling Price per unit,

$\alpha$ : Percentage of on-hand inventory that is lost due to deterioration,

$q: \quad$ Order quantity,

$K \times\left(q^{\gamma-1}\right): \quad$ Ordering cost per cycle where 0 ,

$q^{* *}:$ Modified fuzzy economic ordering / production quantity (FEOQ/FEPQ),

$q^{*}:$ Traditional economic ordering quantity (EOQ),

$\phi(t)$ : On-hand inventory level at time $\mathrm{t}$,

$\Pi_{1}(q)$ : Net profit per unit of producing q units per cycle in crisp strategy,

$\Pi(q)$ : Average profit per unit of producing $\mathrm{q}$ units per cycle in crisp strategy,

$\widetilde{\Pi}_{1}(q, \rho)$ : The net profit per unit per cycle in fuzzy decision space,

$\widetilde{\Pi}(q, \rho)$ : The average profit per unit per cycle in fuzzy decision space,

$\widetilde{h}:$ Fuzzy holding cost per unit,

$\widetilde{K} \times\left(q^{\gamma-1}\right): \quad$ Fuzzy setup cost per cycle. 


\section{Mathematical Model}

Denote $\phi(t)$ as the on-hand inventory level at timet. During a change in time from point $t$ to $t+d t$, where $t+d t>t$, the on-hand inventory drops from $\phi(t)$ to $\phi(t+d t)$. Then $\phi(t+d t)$ is given as:

$$
\phi(t+d t)=\phi(t)-r d t-\alpha \phi(t) d t .
$$

$\phi(t+d t)$ can be rewritten as: $\phi(t+d t)-\frac{\phi(t)}{d t}=-r-\alpha \phi(t)$

and $d t \rightarrow(0), \phi(t+d t)-\frac{\phi(t)}{d t}$ reduces to $\frac{\phi(t)}{d t}+\alpha \phi(t)+r=0$

It is a differential equation, solution is

$$
\phi(t)=-\frac{r}{\alpha}+\left(q+\frac{r}{\alpha}\right) \times \exp -\alpha \times t .
$$

Where $q$ is the order quantity which is instantaneously replenished at the beginning of each cycle of length $t_{c}$ units of time. The stock is replenished by q units each time these units are totally depleted as a result of outside demand and deterioration. Behavior of the inventory level for the above model is illustrated in Fig. 1. The cycle length, $t_{c}$, is determined by first substituting $t_{c}$ into $\phi(t)$ and then setting it equal to zero to get:

$$
t_{c}=\frac{1}{\alpha} \ln \left(\frac{\alpha q+r}{r}\right) .
$$

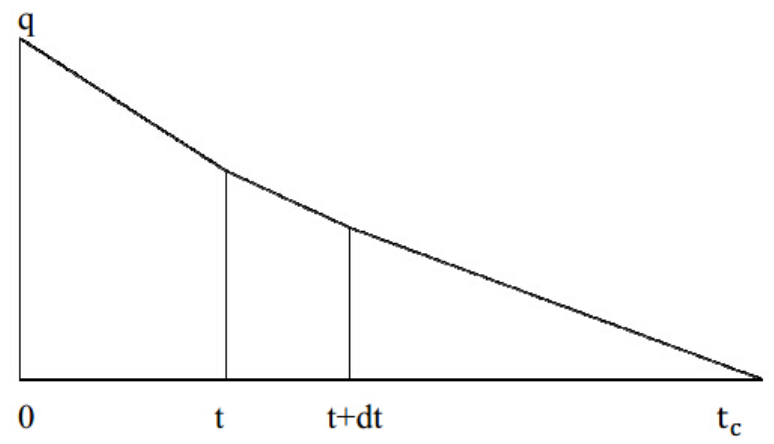

Figure 1. Behavior of the Inventory over a Cycle for a Deteriorating Item

Equation $\phi(t)$ and $t_{c}$ are used to develop the mathematical model. It is worthy to mention that as $\alpha$ approaches to zero, $t_{c}$ approaches to $\frac{q}{r}$. Then the total number of units lost per cycle, $L$, is given as:

$$
L=r\left[\frac{q}{r}-\frac{1}{\alpha} \ln \left(\frac{\alpha q+r}{r}\right)\right]
$$


The total cost per cycle, $T C(q)$, is the sum of the procurement cost per cycle, $K+c q$ and the holding cost per cycle, $H C(q) . H C(q)$ is obtained from equation $\phi(t)$ as:

$$
\begin{aligned}
H C(q) & =\int_{0}^{t_{c}} h \varphi(t) d t \\
& =h \int_{0}^{\frac{1}{\alpha} \ln \left(\frac{\propto q+r}{r}\right)}\left[-\frac{r}{\alpha}+\left(q+\frac{q}{\alpha}\right) \times e^{-\alpha t}\right] d t \\
& =h \times\left[\frac{q}{\propto}-\frac{r}{\alpha^{2}} \ln \left(\frac{\alpha q+r}{r}\right)\right] \\
T C(q)= & \left(K \times q^{\gamma-1}\right)+c q+h \times\left[\frac{q}{\alpha}-\frac{r}{\alpha^{2}} \times \ln \left(\frac{(\alpha q+r)}{r}\right)\right]
\end{aligned}
$$

The total cost per unit of time, $T C U(q)$, is given by dividing equation $T C(q)$ by $t_{c}$ to give:

$$
\begin{aligned}
\operatorname{TCU}(q) & =\left[\left(K \times q^{\gamma-1}\right)+c q+h \times\left[\frac{q}{\alpha}-\frac{r}{\alpha^{2}} \times \ln \left(\frac{(\alpha q+r)}{r}\right)\right]\right] \times\left[\frac{1}{\alpha} \ln \left(\frac{(\alpha q+r)}{r}\right)\right]^{-1} \\
& =\frac{K \propto+(c \propto+h) q}{\ln \left(1+\frac{\alpha q}{r}\right)}-\frac{h r}{\alpha}
\end{aligned}
$$

As $\alpha$ approaches zero in equation $T C U(q)$ reduce to $T C U(q)=\frac{K r}{q}+c r+\frac{h q}{2}$, whose solution is given by the traditional EOQ formula, $q^{*}=\sqrt{ } \frac{2 K r}{h}$. The total profit per cycle is $\Pi_{1}(q)$.

$$
\begin{aligned}
\Pi_{1}(q) & =(q-L) \times P_{s}-T C(q) \\
& =(q-L)\left(\times P_{s}-\left(K \times q^{(\gamma-1)}\right)-c q-h \times\left[\frac{q}{\alpha}-\frac{r}{\alpha^{2}} \times \ln \left(\frac{\alpha q+r}{r}\right)\right]\right.
\end{aligned}
$$

Where $L$, the number of units lost per cycle due to deterioration, and $T C(q)$ the total cost per cycle, are calculated from equations $L$ and $T C(q)$, respectively. The average profit $\Pi(q)$ per unit time is obtained by dividing $t_{c}$ in $\Pi_{1}(q)$. Hence the profit maximization problem is

$$
\left\{\begin{array}{c}
\text { Maximize } \Pi_{1}(q), \\
\forall q \geq 0, \\
\Pi_{1}(q, \rho)=F_{1}(q, \rho)+F_{2}(q, \rho) h+F_{3}(q, \rho) K .
\end{array}\right.
$$

Where

$$
\left\{\begin{array}{l}
\left.F_{1}(q, \rho)=\left(q \times P_{s}\right)-c q-K_{1}(\rho-1)^{2} r^{\alpha_{1}}\right) \\
F_{2}(q, p)=-\left[\frac{q^{2}}{2 r \rho}\right] \\
F_{3}(q, p)=-1
\end{array}\right.
$$

Stat., Optim. Inf. Comput. Vol. 2, September 2014. 


\section{Fuzzy Mathematical Model}

The holding cost and ordering cost are replaced by fuzzy numbers $\widetilde{h}$ and $\widetilde{K}$ respectively. By expressing $\widetilde{h}$ and $\widetilde{K}$ as the normal triangular fuzzy numbers $\left(h_{1}, h_{0}, h_{2}\right)$ and $\left(K_{1}, K_{0}, K_{2}\right)$, where $h_{1}=h-\Delta_{1}, h_{0}=h, h_{2}=h+$ $\Delta_{2}, K_{1}=K-\Delta_{2}, K_{0}=K, K_{2}=K+\Delta_{4}$ such that $0<\Delta_{1}<h, 0<\Delta_{2}, 0<$ $\Delta_{3}<K, 0<\Delta_{4}, \Delta_{1}, \Delta_{2}, \Delta_{3}$ and $\Delta_{4}$ are determined by the decision maker based on the uncertainty of the problem.

The membership function of fuzzy holding cost and fuzzy ordering cost are considered as:

$$
\begin{array}{r}
\mu_{\widetilde{h}}(h)= \begin{cases}\frac{h-h_{1}}{h_{0}-h_{1}}, & h_{1} \leq h \leq h_{0} \\
\frac{h_{2}-h}{h_{2}-h_{0}}, & h_{1} \leq h \leq h_{0} \\
0, & \text { otherwise }\end{cases} \\
\mu_{\widetilde{k}}(h)= \begin{cases}\frac{K-K_{1}}{K_{0}-h_{1}}, & K_{1} \leq K \leq K_{0} \\
\frac{K_{2}-K}{K_{2}-K_{0}}, & K_{0} \leq K \leq K_{2} \\
0, & \text { otherwise }\end{cases}
\end{array}
$$

Then the centroid for $\widetilde{h}$ and $\widetilde{K}$ are given by:

$\left.M_{\widetilde{h}}=\frac{\left(h_{1}+h_{0}+h_{2}\right)}{3}=h+\frac{\left(\Delta_{2}-\Delta_{1}\right)}{3}\right), M_{\widetilde{k}}=\frac{K_{1}+K_{0}+K_{2}}{3}=K+\left(\Delta_{4}-\Delta_{3}\right) / 3$.

For fixed values of $\mathrm{q}$ and $\rho$, let $\Pi_{1}(h, K)=F_{1}(q)+F_{2}(q) h+F_{3}(q) K=y$, $h=\frac{y-F_{1}-F_{3} K}{F_{2}}, \frac{\Delta_{2}-\Delta_{1}}{3}=\psi$, and $\frac{\Delta_{4}-\Delta_{3}}{3}=\psi_{2}$.

By extension principle the membership function of the fuzzy profit function is given by

$$
\begin{aligned}
\mu_{\Pi_{1}(\widetilde{h}, \widetilde{k})}(y) & =\sup _{(h, k) \epsilon \Pi_{1}^{-1}(y)}\left\{\mu_{\widetilde{h}} \wedge \mu_{\widetilde{k}(K)}\right\} \\
& =\sup _{k_{1} \leq k \leq k_{2}}\left\{\mu_{\widetilde{h}}\left(\frac{y-F_{1}-F_{3} K}{F_{2}}\right) \wedge \mu_{\widetilde{k}(K)}\right\}
\end{aligned}
$$

Now,

$$
\mu_{\tilde{h}}\left(\frac{y-F_{1}-F_{3} K}{F_{2}}\right)= \begin{cases}\frac{y-F_{1}-F_{2} h_{1}-F_{3} K}{F_{2}\left(h_{0}-h_{1}\right)}, & u_{2} h_{1} \leq K \leq u_{1} \\ \frac{F_{1}+F_{2} h_{2}+F_{3} K-y}{F_{2}\left(h_{2}-h_{0}\right)}, & u_{3} \leq K \leq u_{2} \\ 0, & \text { otherwise }\end{cases}
$$

where $u_{1}=\frac{y-F_{1}-F_{2} h_{1}}{F_{3}}, u_{2}=\frac{y-F_{1}-F_{2} h_{0}}{F_{3}}$ and $u_{3}=\frac{y-F_{1}-F_{2} h_{2}}{F_{3}}$.

Fig. 2 exhibits the graph of $\mu_{\widetilde{h}}\left(\frac{y-F_{1}-F_{3} K}{F_{2}}\right)$ and $\mu_{\widetilde{h}}$ when $u_{2} \leq K$ and $K \leq u_{1}$ 


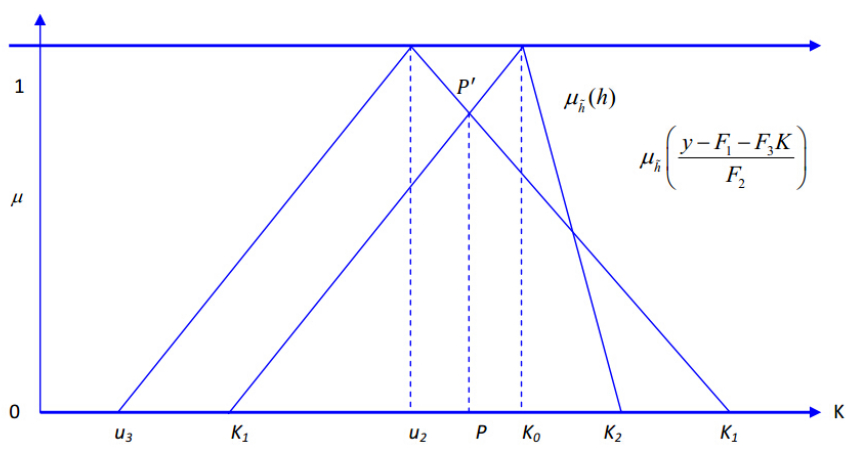

Figure 2. Defuzzification by using Centroid Method

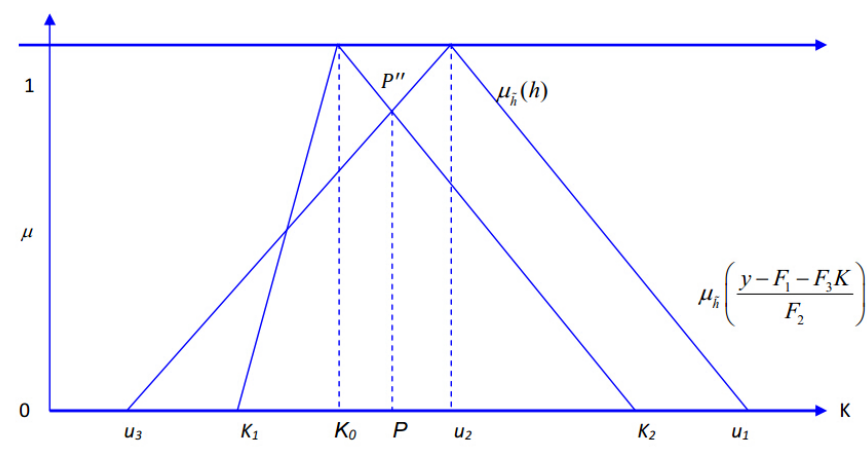

Figure 3. Defuzzification by using Centroid Method

then $y \leq F_{1}+F_{2} h_{0}+F_{3} K_{0}$ and $y \geq F_{1}+F_{2} h_{1}+F_{3} K_{1}$. It is clear that for every $y \epsilon\left[F_{1}+F_{2} h_{1}+F_{3} K_{1}, F_{1}+F_{2} h_{0}+F_{3} K_{0}\right], \mu_{y}(Y)=P P^{\prime}$. From the $\mu_{\widetilde{h}}$ and $\mu_{\widetilde{h}}\left(\frac{y-F_{1}-F_{3} K}{F_{2}}\right)$ the value of $P P^{\prime}$ may be found by solving the following equation :

$\frac{K-K_{1}}{K_{0}-K_{1}}=\frac{y-F_{1}+F_{2} h_{1}-F_{3} K}{F_{2}\left(h_{0}-h_{1}\right)}$ or $K=\frac{\left(y-F_{1}-F_{2} h_{1}\right)\left(K_{0}-K_{1}\right)+F_{2} K_{1}\left(h_{0}-h_{1}\right)}{F_{2}\left(h_{0}-h_{1}\right)+F_{3}\left(K_{0}-K_{1}\right)}$.

Therefore,

$$
P P^{\prime}=\frac{K-K_{1}}{K_{0}-K_{1}}=\frac{y-F_{1}-F_{2} h_{1}-F_{3} K}{F_{2}\left(h_{0}-h_{1}\right)+F_{3}\left(K_{0}-K_{1}\right)}=\mu_{1}(y), \text { (say). }
$$

Fig. 3 exhibits the graph of $\mu_{\widetilde{h}}\left(\frac{y-F_{1}-F_{3} K}{F_{2}}\right)$ and $\mu_{\widetilde{h}}$ when $u_{3} \leq K$ and $K \leq$ $u_{2}$ then $y \leq F_{1}+F_{2} h_{2}+F_{3} K_{2}$ and $y \geq F_{1}+F_{2} h_{0}+F_{3} K_{0}$. It is evident that for every $y \epsilon\left[F_{1}+F_{2} h_{0}+F_{3} K_{0}, F_{1}+F_{2} h_{2}+F_{3} K_{2}\right], \mu_{\widetilde{y}}(y)=P P^{\prime}$. From the $\mu_{\widetilde{h}}$ and $\mu_{\widetilde{h}}\left(\frac{y-F_{1}-F_{3} K}{F_{2}}\right)$ the value of $P P^{\prime}$ may be found by solving the following equation :

$\frac{K_{2}-K}{K_{2}-K_{0}}=F_{1}+F_{2} h_{2}+\frac{F_{3} K-y}{F_{2}\left(h_{2}-h_{0}\right)}$ or $K=\frac{F_{2} K_{2}\left(h_{2}-h_{0}\right)-\left(F_{1}+F_{2} h_{2}-y\right)\left(K_{2}-K_{0}\right)}{F_{2}\left(h_{2}-h_{0}\right)+F_{3}\left(K_{2}-K_{0}\right)}$.

Stat., Optim. Inf. Comput. Vol. 2, September 2014. 
Therefore,

$$
P P^{\prime}=\frac{K_{2}-K}{K_{2}-K_{0}}=F_{1}+F_{2} h_{2}+F_{3} K_{2}-y / F_{2}\left(h_{2}-h_{0}\right)+F_{3}\left(K_{2}-K_{0}\right)=\mu_{2}(y), \text { (say). }
$$

Thus the membership function for fuzzy total profit is given by:

$$
\mu_{\Pi_{1}(\widetilde{h}, \widetilde{k})}= \begin{cases}\mu_{1} y, & F_{1}+F_{2} h_{1}+F_{3} K_{1} \leq y \leq F_{1}+F_{2} h_{0}+F_{3} K_{0} \\ \mu_{2} y, & F_{1}+F_{2} h_{0}+F_{3} K_{0} \leq y \leq F_{1}+F_{2} h_{2}+F_{3} K_{2} \\ 0, & \text { otherwise }\end{cases}
$$

Now, let $P_{1}=\int_{-\infty}^{\infty} \mu_{\Pi_{1}(\widetilde{h}, \widetilde{k})}(y) d y$ and $R_{1}=\int_{-\infty}^{\infty} y \mu_{\Pi_{1}(\widetilde{h}, \widetilde{k})}(y) d y$

Hence, the centroid for fuzzy total profit is given by

$$
\begin{aligned}
& \widetilde{\Pi}_{1}(q)=M_{\widetilde{T P}(q)}=\frac{R_{1}}{P_{1}}=F_{1}(q)+F_{2}(q) h+F_{3}(q) K+\Psi_{1} F_{2}(q)+\Psi_{2} F_{2}(q) \\
& \widetilde{\Pi}_{1}(q)=M_{\widetilde{T} P}(q)=F_{1}+\left(h+\Psi_{1}\right) F_{2}+\left(K+\Psi_{2}\right) F_{3}
\end{aligned}
$$

where, $F_{1}(q), F_{2}(q)$ and $F_{3}(q)$ are given by the equations.

Hence the profit Maximization problem is

$$
\text { Maximize } \widetilde{\Pi}_{1}(q)=M_{\widetilde{T} P}(q), \quad \forall q \geq 0
$$

\section{Optimization}

The optimal ordering quantity q per cycle can be determined by differentiating equation $\widetilde{\Pi}_{1}(q)$ with respect to q, then setting these to zero.

In order to show the uniqueness of the solution in, it is sufficient to show that the net profit function throughout the cycle is concave in terms of ordering quantity q. The second order derivates of equation $\widetilde{\Pi}_{1}(q)$ with respect to $q$ are strictly negative. Consider the following proposition.

Proposition 1 : The net profit $\widetilde{\Pi}_{1}(q)$ per cycle is concave in q. Conditions for optimal $q$

$$
\frac{d \widetilde{\Pi}_{1}(q)}{d q}=\frac{r}{(\alpha q+r)}\left(P_{s}+\frac{\left(h+\psi_{1}\right)}{\alpha}\right)-\left(\left(K+\psi_{2}\right)(\gamma-1) q^{\gamma-2}+c+\frac{\left(h+\psi_{1}\right)}{\alpha}\right)=0
$$

The second order derivative of the net profit per cycle with respect to q can be expressed as:

$$
\frac{d^{2} \widetilde{\Pi}_{1}(q)}{d q^{2}}=\frac{-r}{(\alpha q+r)^{2}}\left(P_{s} \alpha+\left(h+\psi_{1}\right)\right)-\left(K+\psi_{2}\right)(\gamma-1)(\gamma-2) q^{\gamma-3}
$$


Table II. Optimal Values of the Proposed Model

\begin{tabular}{|c|c|c|c|c|c|c|c|}
\hline Model & Iteration & $t^{*}$ & $L^{*}$ & $q$ & Dynamic OC & $\widetilde{\Pi}_{1}(q)$ & $\widetilde{\Pi}_{(q)}$ \\
\hline Fuzzy & 78 & 2.354408 & 144.1828 & 2498.591 & 4.002448 & 30002.35 & 12743.03 \\
\hline Crisp & 39 & 2.355722 & 173.2163 & 3000.082 & 4.06 & 36024.74 & 15292.44113 \\
\hline \% Change & - & 0.11943 & 20.13659 & 20.07095 & 1.4379 & 20.0731 & 20.0063 \\
\hline Crisp & 29 & 5.000043 & - & 6000.052 & 2.59 & 74997.42 & 14999.35501 \\
\hline \% Change & - & 112.369 & - & 140.1374 & 35.2896 & 149.9718 & 17.7064 \\
\hline
\end{tabular}

Since, $r>0$ and $P_{s} \alpha+\left(h+\psi_{1}\right)>0,\left(K+\Psi_{2}\right)>0, q>0$ and $0<\gamma<1$ so the equation $d^{2} \widetilde{\Pi}_{1}(q) / d q^{2}$ is negative.

Proposition 1 shows that the second order derivative of equation $\widetilde{\Pi}_{1}(q)$ with respect to $q$ are strictly negative.

The objective is to determine the optimal values of $q$ to maximize the unit profit function of $\widetilde{\Pi}_{1}(q)$. It is very difficult to derive the optimal values of $q$, hence unit profit function. There are several methods to cope with constraints optimization problem numerically. But here LINGO 13.0 software is used to derive the optimal values of the decision variables.

\section{Numerical Example}

Consider an inventory situation where $K$ is Rs. 200 per order, $h$ is Rs.5 per unit per unit of time, $r$ is 1000 units per unit of time, $c$ is Rs. 100 per unit, the selling price per unit $P_{s}$ is Rs.125, $\gamma$ is $0.5, \Delta_{1}=0.002, \Delta_{2}=0.02, \Delta_{3}=0.002$, and $\Delta_{4}=0.2$ and $\alpha$ is 0.05 . The optimal solution that maximizes equation $\widetilde{\Pi}_{1}(q)$ and $q^{* *}$ and $q^{*}$ are determined by using LINGO 13.0 version software and the results are tabulated in Table II.

\section{Sensitivity Analysis}

It is interesting to investigate the influence of $\alpha$ on retailer behavior. The computational results shown in Table III indicates the following managerial phenomena: when the percentage of on-hand inventory that is lost due to deterioration $\alpha$ increases, the replenishment cycle length, the optimal replenishment quantity, the optimal total number of units lost per cycle and optimal net profit per unit per cycle decrease respectively. The fuzzy average profit is insensitive but variable ordering cost is fluctuating with increasing in the percentage value of the major parameter $\alpha$. Fig. 4 represents the relationship between the order quantity $q$ and dynamic setup cost OC. Fig. 5 shows the relationship between the order quantity $q$ and units lost per cycle due to deterioration $L$ and Fig. 6 represents the three dimensional mesh plot of units lost per cycle due to deterioration $L$, order quantity $q$ and net profit per cycle $\widetilde{\Pi}_{1}(q)$. 


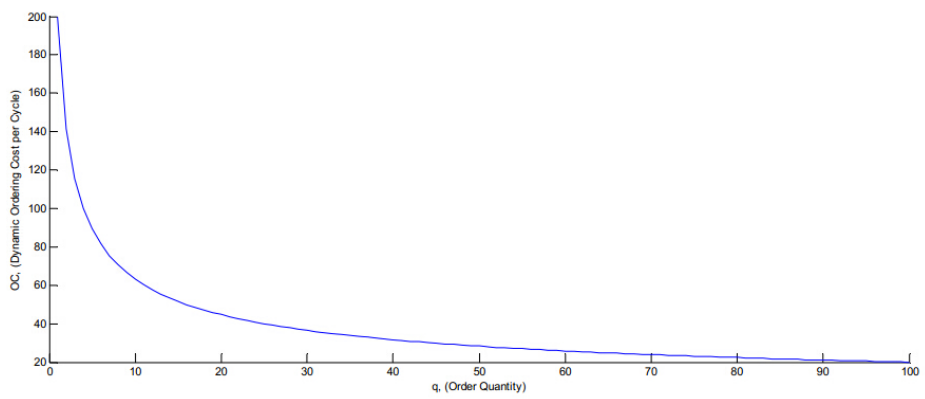

Figure 4. Two Dimensional Plot of Order Quantity, q and Dynamic Ordering Cost, OC

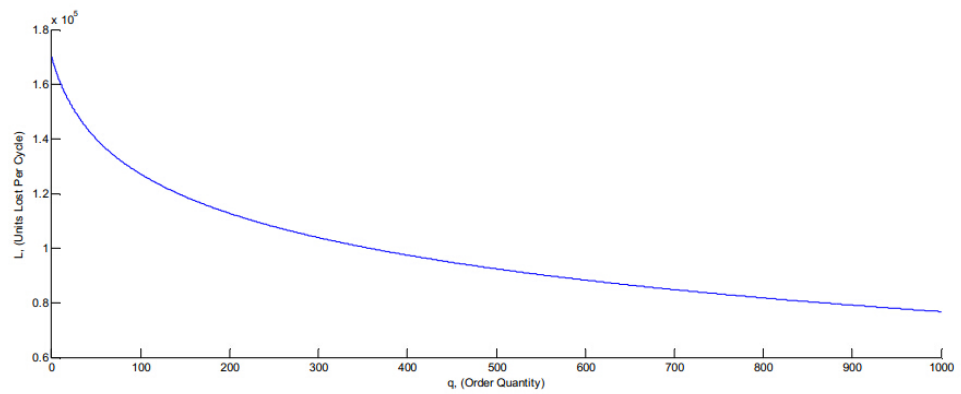

Figure 5. Two Dimensional Plot of Order Quantity q and Units Lost per Cycle L

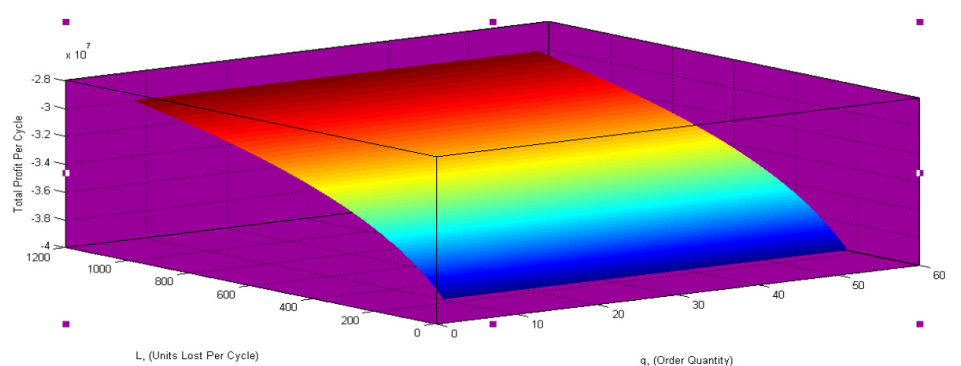

Figure 6. Three Dimensional Mesh Plot of Order Quantity q, Units Lost per Cycle L and Fuzzy Net Profit per Cycle $\widetilde{\Pi}_{1}(q)$

Table III. Sensitivity Analysis of $\alpha$

\begin{tabular}{|c|c|c|c|c|c|c|c|}
\hline$\alpha$ & Iteration & $t^{*}$ & $L^{*}$ & $q^{* *}$ & $O C$ & $\Pi_{1}(q)$ & $\Pi(q)$ \\
\hline .04 & 79 & 2.632423 & 143.5883 & 2776.012 & 3.797193 & 33477.87 & 12717.51 \\
\hline .10 & 77 & 1.541034 & 125.0818 & 1666.115 & 4.901408 & 19751.28 & 12816.91 \\
\hline .12 & 76 & 1.353988 & 116.2044 & 1470.192 & 5.217786 & 17376.37 & 12833.48 \\
\hline .30 & 73 & 0.6472356 & 67.10947 & 714.3450 & 7.485473 & 8342.624 & 12889.62 \\
\hline .60 & 71 & 0.3462466 & 38.59017 & 384.8308 & 10.19855 & 4464.829 & 12895.16 \\
\hline .90 & 71 & 0.2364122 & 27.03369 & 263.4459 & 12.32615 & 3044.243 & 12876.84 \\
\hline
\end{tabular}




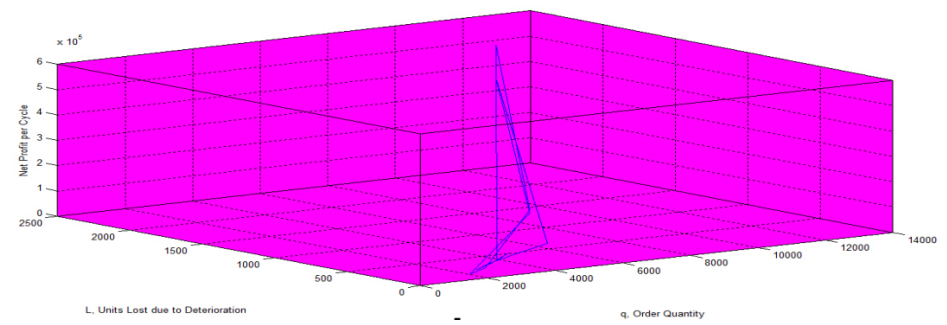

Figure 7. Sensitivity Plotting of Order Quantity q, Units Lost per Cycle L and Fuzzy Net Profit per Cycle $\widetilde{\Pi}_{1}(q)$

It is interesting to investigate the influence of the major parameters $\widetilde{K}, \widetilde{h}, \mathrm{r}, \mathrm{c}, P_{s}$ and $\gamma$ on retailer's behavior. The computational results shown in Table IV indicate the following managerial phenomena:

- $t_{c}$ the replenishment cycle length, $\mathrm{q}$ the optimal replenishment quantity, $L$ number of units lost due to deterioration, $\widetilde{\Pi}_{1}$ the optimal net profit per unit per cycle and $\widetilde{\Pi}$ the optimal average profit per unit per cycle are insensitive to the parameter $\widetilde{K}$ but OC variable setup cost is sensitive to the parameter $\widetilde{K}$.

- $t_{c}$ the replenishment cycle length, $q$ the optimal replenishment quantity, $L$ number of units lost due to deterioration, OC variable setup cost $\widetilde{\Pi}_{1}$ the optimal net profit per unit per cycle are moderately sensitive to the parameter $\widetilde{h}$ but $\widetilde{\Pi}_{1}$ the optimal average profit per unit per cycle is insensitive to the parameter $\widetilde{h}$.

- $q$ the optimal replenishment quantity, $L$ number of units lost due to deterioration, $\mathrm{OC}$ variable setup cost, $\widetilde{\Pi}_{1}$ the optimal net profit per unit per cycle and $\widetilde{\Pi}$ the optimal average profit per unit per cycle are sensitive to the parameter $r$ but $t_{c}$ the replenishment cycle length is insensitive to the parameter $r$.

- $t_{c}$ the replenishment cycle length, $L$ number of units lost due to deterioration, $q$ the optimal replenishment quantity, OC variable setup cost, $\widetilde{\Pi}_{1}$ the optimal net profit per unit per cycle and $\widetilde{\Pi}$ the optimal average profit per unit per cycle are sensitive to both the parameters $c$ and $P_{s}$.

- $t_{c}$ the replenishment cycle length, $L$ number of units lost due to deterioration, $q$ the optimal replenishment quantity, $\widetilde{\Pi}_{1}$ the optimal net profit per unit per cycle and $\widetilde{\Pi}$ the optimal average profit per unit per cycle are insensitive to the parameter $\gamma$ but OC variable setup cost is sensitive to the parameter $\gamma$. 
Table IV. Sensitivity Analyses of the parameters $\widetilde{K}, \widetilde{h}, r, c, P_{s}$, and $\gamma$.

\begin{tabular}{|c|c|c|c|c|c|c|c|c|}
\hline Parameter & Value & Iteration & $t^{*}$ & $L^{*}$ & $q^{*}$ & $\mathrm{OC}$ & $\widehat{\Pi}_{1}(q)$ & $\widetilde{\Pi}(q)$ \\
\hline \multirow{3}{*}{$\tilde{K}$} & 150 & 80 & 2.353388 & 144.1803 & 2498.568 & 3.002180 & 300003.35 & 12743.59 \\
\hline & 250 & 82 & 2.354428 & 144.1853 & 2498.613 & 5.002707 & 30001.35 & 12742.52 \\
\hline & 500 & 96 & 2.354528 & 144.1978 & 2498.726 & 10.00387 & 29996.35 & 12739.85 \\
\hline \multirow{3}{*}{$\tilde{h}$} & 3 & 80 & 2.901686 & 221.0546 & 3122.741 & 3.580184 & 37143.31 & 12800.60 \\
\hline & 6 & 88 & 2.151585 & 119.9971 & 2271.582 & 4.197678 & 27371.65 & 12721.62 \\
\hline & 10 & 90 & 1.600337 & 65.76943 & 1666.106 & 4.901420 & 20264.8 & 12662.84 \\
\hline \multirow{3}{*}{$\mathrm{r}$} & 1050 & 64 & 2.354403 & 151.3912 & 2623.514 & 3.905994 & 31502.76 & 13380.36 \\
\hline & 1500 & 70 & 2.354372 & 216.2673 & 3747.825 & 3.268012 & 45006.26 & 19116.04 \\
\hline & 2000 & 70 & 2.354356 & 288.3526 & 4997.065 & 2.830191 & 60009.87 & 25488.86 \\
\hline \multirow{3}{*}{$\mathrm{c}$} & 105 & 67 & 1.860882 & 89.32065 & 1950.202 & 4.530368 & 18891.65 & 10151.99 \\
\hline & 110 & 70 & 1.379272 & 48.67222 & 1427.945 & 5.294410 & 10456.78 & 7581.377 \\
\hline & 112 & 63 & 1.189849 & 36.10594 & 1225.954 & 5.713946 & 7803.526 & 6558.420 \\
\hline \multirow{3}{*}{$P_{S}$} & 120 & 78 & 1.905225 & 93.69859 & 1998.924 & 4.474816 & 19349.07 & 10155.79 \\
\hline & 150 & 80 & 4.460501 & 536.5365 & 4997.037 & 2.830199 & 115650.5 & 25927.7 \\
\hline & 200 & 79 & 8.105314 & 1888.704 & 9994.019 & 2.001259 & 432561.9 & 53367.69 \\
\hline \multirow{3}{*}{$\gamma$} & 0.3 & 75 & 2.354352 & 144.1757 & 2498.527 & 0.8371329 & 30005.51 & 12744.7 \\
\hline & 0.6 & 82 & 2.354468 & 144.1903 & 2498.658 & 8.751662 & 29997.6 & 12740.71 \\
\hline & 0.9 & 90 & 2.354694 & 144.2185 & 2498.913 & 91.49517 & 29914.85 & 12704.35 \\
\hline
\end{tabular}

\section{Conclusion}

In a modified FEOQ model with a percentage of the on-hand inventory lost due to deterioration and variable ordering cost as characteristic features and the inventory conditions govern the item stocked. This model provides a useful property for finding the optimal profit and ordering quantity with deteriorated units of lost sales. A new fuzzy mathematical model with variable setup cost is developed and compared to the traditional EOQ model numerically. The economic order quantity, $q^{* *}$ for modified model is found to be less than that of the traditional, $q^{*}$, i.e. $q^{* *}<q^{*}$. Finally, wasting the percentage of on-hand inventory due to deterioration effect was demonstrated numerically to have an adverse effect on the average profit per unit per cycle. Hence the utilization of units lost due to deterioration makes the scope of the applications broader. Further, a numerical example is presented to illustrate the theoretical results, and some observations are obtained from sensitivity analysis with respect to the major parameters $\alpha, \widetilde{K}, \widetilde{h}, \mathrm{r}$, c, $P_{s}$ and $\gamma$. The model in this study is a general framework that considers wasting/ none wasting the percentage of on-hand inventory due to deterioration and variable ordering cost simultaneously in fuzzy environment. To the best of its knowledge, this is the model that investigates the impact of units lost due to deterioration and variable ordering cost simultaneously on fuzzy-type environment. In the future study, it is hoped to further incorporate the proposed models into several situations such as shortages are allowed and the consideration of multi-item problem in uncertain nonrandom space. Furthermore, it may also take partial backlogging into account when determining the optimal replenishment policy.

\section{REFERENCES}

Stat., Optim. Inf. Comput. Vol. 2, September 2014. 
1. Bose, S., Goswami, A. and Chaudhuri, K.S. "An EOQ model for deteriorating items with linear time-dependent demand rate and shortages under inflation and time discounting". Journal of Operational Research Society, 46: 775-782, 1995.

2. Goyal, S.K. and Giri, B.C. "Recent trends in modeling of deteriorating inventory'. European Journal of Operational Research, 134: 1-16, 2001.

3. Goyal, S.K. and Gunasekaran, A. "An integrated production-inventory-marketing model for deteriorating items". Computers and Industrial Engineering, 28: 755-762, 1995.

4. Gupta, D. and Gerchak, Y. "Joint product durability and lot sizing models". European Journal of Operational Research, 84: 371-384, 1995.

5. Hariga, M. "An EOQ model for deteriorating items with shortages and time-varying demand". Journal of Operational Research Society, 46: 398-404, 1995.

6. Hariga, M. "An EOQ model for deteriorating items with time-varying demand". Journal of Operational Research Society, 47: 1228-1246, 1996.

7. Hariga, M., "Economic analysis of dynamic inventory models with non-stationary costs and demand". International Journal of Production Economics, 36: 255-266, 1994.

8. Jain, K. and Silver, E. "A lot sizing for a product subject to obsolescence or perishability". European Journal of Operational Research, 75: 287-295, 1994.

9. Mahata, G.C. and Goswami, A. "Production lot size model with fuzzy production rate and fuzzy demand rate for deteriorating item under permissible delay in payments". Journal of Operational Research Society India, 43: 359-375, 2006.

10. Mishra, V.K. "Inventory model for time dependent holding cost and deterioration with salvage value and shortages". The Journal of Mathematics and Computer Science, 4(1): 37-47, 2012.

11. Osteryoung, J.S., Mc Carty D.E. and Reinhart W.L. "Use of EOQ models for inventory analysis". Production and Inventory Management, 3rd Qtr: 39-45, 1986.

12. Padmanabhan, G. and Vrat, P. "EOQ models for perishable items under stock dependent selling rate". European Journal of Operational Research, 86: 281-292, 1995.

13. Pattnaik, M. "Fuzzy NLP for a Single Item EOQ Model with Demand - Dependent Unit Price and Variable Setup Cost". World Journal of Modeling and Simulations, 9(1): 74-80, 2013.

14. Pattnaik, M. "Models of Inventory Control". Lambart Academic Publishing, Germany, 2012.

15. Raafat, F. "Survey of literature on continuously deteriorating inventory models". Journal of Operational Research Society, 42: 89-94, 1991.

16. Roy, T.K. and Maiti, M. "A Fuzzy EOQ model with demand dependent unit cost under limited storage capacity”. European Journal of Operational Research, 99: 425 - 432, 1997.

17. Sahoo, P.K. and Pattnaik, M. "An article "Decision Making Approach to Fuzzy Linear Programming (FLP) Problems with Post Optimal Analysis". International Journal of Operations Research and Information Systems, in press, 2013.

18. Salameh, M.K., Jaber, M.Y. and Noueihed, N. "Effect of deteriorating items on the instantaneous replenishment model". Production Planning and Control, 10(2): 175-180, 1993.

19. Shah, N. "Literature survey on inventory models for deteriorating items". Economics Annals, 44: 221-237, 2000.

20. Tripathy, P.K. and Pattnaik, M. "Fuzzy Supplier Selection Strategies in Supply Chain Management”. International Journal of Supply Chain Management, 2(1): 30-39, 2013.

21. Tripathy, P.K. and Pattnaik, M. "Optimal disposal mechanism with fuzzy system cost under flexibility and Reliability criteria in non-random optimization environment”. Appllied Mathematical Sciences, 3(37): 1823-1847, 2009.

22. Tripathy, P.K. and Pattnaik, M. "Optimal inventory policy with reliability consideration and instantaneous receipt under imperfect production process". International Journal of Management Sciences and Engineering Management, 6(6): 412-420, 2011.

23. Tripathy, P.K., Pattnaik, M. and Tripathy, P. "Optimal EOQ Model for Deteriorating Items with Promotional Effort Cost”. American Journal of Operations Research, 2(2): 260-265, 2012.

24. Tripathy, P.K., Tripathy, P. and Pattnaik, M. "A Fuzzy EOQ Model with Reliability and Demand-dependent Unit Cost". International Journal of Contemporary Mathematical Sciences, 6(30): 1467-1482, 2011.

25. Tsao, Y.C. and Sheen, G.J. "Dynamic pricing, promotion and replenishment policies for a deteriorating item under permissible delay in payment". Computers and Operations Research, 
35: 3562-3580, 2008.

26. Vujosevic, M., Petrovic, D. and Petrovic, R. "EOQ formula when inventory cost is fuzzy". International Journal of Production Economics, 45: 499-504, 1996.

27. Waters, C.D.J. "Inventory Control and Management". (Chichester: Wiley), 1994. 